hep-th/9510183

preprint UT-728

October, 1995

\title{
Prepotentials of $N=2$ Supersymmetric Gauge Theories and Soliton Equations
}

\author{
Tohru Eguchi \\ Department of Physics \\ Faculty of Science \\ University of Tokyo \\ Tokyo 113, Japan \\ and \\ Sung-Kil Yang \\ Institute of Physics \\ University of Tsukuba \\ Ibaraki 305, Japan
}

\begin{abstract}
Using recently proposed soliton equations we derive a basic identity for the scaling violation of $N=2$ supersymmetric gauge theories $\sum_{i} a_{i} \partial F / \partial a_{i}-2 F=8 \pi i b_{1} u$. Here $F$ is the prepotential, $a_{i}$ 's are the expectation values of the scalar fields in the vector multiplet, $u=$ $1 / 2 \operatorname{Tr}\left\langle\phi^{2}\right\rangle$ and $b_{1}$ is the coefficient of the one-loop $\beta$-function. This equation holds in the Coulomb branch of all $N=2$ supersymmetric gauge theories coupled with massless matter.
\end{abstract}


Recently there have been some major progress in our understanding of the non-perturbative strong coupling behavior of 4 dimensional supersymmetric gauge theories and string theories in various dimensions based on the idea of $\mathrm{S}$ (strong-weak) duality [1]-[13]. In the case of $N=2$ supersymmetric gauge fields for various gauge groups, many of their low energy effective Lagrangians have been determined exactly with or without the presence of matter fields [14]-22]. There now exist a considerable amount of data for the algebraic curves and differential forms which are used to compute the prepotentials of the $N=2$ effective Lagrangian.

Quite recently an idea has been proposed which may possibly organize these data within the framework of some known integrable systems 24[26]. In this article we will adopt a machinery from the soliton theory and derive an important relation obeyed by the prepotentials $F$ of general $N=2$ supersymmetric gauge theories coupled to massless matter fields

$$
\sum_{i=1}^{r} a_{i} \frac{\partial F}{\partial a_{i}}-2 F=8 \pi i b_{1} u
$$

Here $b_{1}$ is the coefficient of the one-loop $\beta$-function and $a_{i}(i=1, \cdots, r)$ are the expectation values of the Cartan components of the scalar fields in the vector multiplet. $r$ is the rank of the gauge group $G$ and $u=1 / 2 \operatorname{Tr}\left\langle\phi^{2}\right\rangle$. Eq.(1) holds in the Coulomb branch of the theory. The right-hand-side of the above equation shows precisely the amount of the scaling violation dictated by the $\beta$-function.

The simplest version of the formula (1) was obtained previously by Matone for the case $G=S U(2), N_{f}=0$ making use of the Picard-Fuchs (P-F) equation [27]. In the case of the $S U(2)$ theory it is easy to generalize the analysis to the case of matter fields $N_{f} \neq 0$. The method of P-F equations, however, becomes messy and complicated when we go to higher rank groups and we need an alternative method of derivation. As we shall see, the machinery of soliton equation is particularly suited to our purpose and it is easy to derive the formula (11).

During the preparation of this article there appeared a new preprint "On the Relation Between the Holomorphic Prepotentials and the Quantum Moduli in SUSY Gauge Theories" (hep-th/9510129) by J. Sonnenschein, S. Theisen and S. Yankielowicz [28] which proves (1) from a somewhat different starting point. 
Let us first recall the $S U(2)$ case without matter fields. The curve is given by $y^{2}=\left(x^{2}-\Lambda^{4}\right)(x-u)[2]$ and the period integral of the meromorphic 1-form $\lambda=\frac{\sqrt{2}}{2 \pi} \frac{(x-u)}{y} d x$ obeys the P-F equation 29, 30]

$$
\frac{d^{2} \omega(u)}{d u^{2}}+\frac{1}{4\left(u^{2}-\Lambda^{4}\right)} \omega(u)=0 .
$$

Since eq.(2) has no first-derivative term, the Wronskian made out of its solutions $a(u), a_{D}(u)$ is $u$-independent

$$
a(u) a_{D}(u)^{\prime}-a_{D}(u) a(u)^{\prime}=\text { constant. }
$$

(Note that the Wronskian is invariant under the $S L(2, R)$ transformation among $a$ and $a_{D}$.) By integrating (3) over $u$ we find

$$
a(u) \frac{\partial F}{\partial a(u)}-2 F(u)=\text { constant } \times u .
$$

Recall that $a_{D}=\partial F / \partial a$. Evaluating both sides of (4) at $u=\infty$ by making use of the weak-coupling expansion

$$
\begin{aligned}
& F \approx \frac{i}{2 \pi} a^{2}\left(\log a^{2} / \Lambda^{2}+O\left((\Lambda / a)^{4}\right)\right), \\
& u \approx \frac{1}{2} a^{2}\left(1+O\left((\Lambda / a)^{4}\right)\right)
\end{aligned}
$$

we find the value of the constant $=4 i / 2 \pi$. In fact this value is proportional to the coefficient of the $a^{2} \log a^{2}$ term in $F$ and hence the one-loop $\beta$ function.

It is straightforward to generalize the above computation to the $N_{f} \neq 0$ cases $\left(N_{f}=1,2,3\right)$. We find that the first-derivative term is always absent in the P-F equation [31] and hence the Wronskian is a constant proportional to $b_{1}=\left(4-N_{f}\right) / 16 \pi^{2}$.

We recall that in $N=2$ supersymmetric Yang-Mills theories with the general gauge group, one introduces a hyperelliptic curve $\Sigma$ and a meromorphic differential $\lambda$ whose periods give expectation values $a_{i}, a_{i}^{D}(i=1, \cdots, r)$ [14, 15]. The meromorphic 1 -form $\lambda$ has a double pole at $\infty$ whose residue is proportional to $b_{1}$. When one couples massive matter, $\lambda$ may also have a simple pole at $\infty$ whose residue is a sum of the masses $m_{i}$ with half-integer coefficients. P-F equations in the case of higher rank groups are partial differential equations and their analysis seems to involve a number of technical 
complications. In the following we, instead, use an approach based on soliton theory, i.e. Whitham's method of adiabatic perturbation of integrable systems [23].

Let us now describe in some detail the structure of the Whitham dynamics which has been proposed to be relevant for the analysis of $N=2$ supersymmetric gauge theories [24]-26]. We follow the presentation of ref.[26]. In [24, 26] new "time" variables $T_{n}(n=0,1, \cdots)$ are introduced which are coupled to the $(n+1)$-th order pole of the differential $\lambda$. It is assumed that $\lambda$ satisfies the following equations

$$
\frac{\partial \lambda}{\partial a_{i}}=\omega_{i}(i=1, \cdots, r), \quad \frac{\partial \lambda}{\partial T_{0}}=\Omega_{0}, \quad \frac{\partial \lambda}{\partial T_{n}}=\Omega_{n}(n=1,2, \cdots) .
$$

Here $\omega_{i}$ are holomorphic differentials normalized as $\oint_{\alpha_{j}} \omega_{i}=\delta_{i j} . \Omega_{n}, \Omega_{0}$ are meromorphic differentials of the 2 nd and 3rd kind, respectively, with a behavior $\Omega_{n} \approx-n z^{-n-1}, \quad \Omega_{0} \approx z^{-1}$ at $x=1 / z=\infty$. $\Omega_{n}$ and $\Omega_{0}$ have vanishing periods for the $\alpha$-cycles. $\left(\Omega_{0}\right.$ behaves as $\Omega_{0} \approx-z_{*}^{-1}$ at $x=1 / z_{*}=$ $\infty$ on the other Riemann sheet of the hyperelliptic surface.) Eq.(7) leads to the integrability conditions for the differentials

$$
\frac{\partial \omega_{j}}{\partial a_{i}}=\frac{\partial \omega_{i}}{\partial a_{j}}, \quad \frac{\partial \omega_{i}}{\partial T_{n}}=\frac{\partial \Omega_{n}}{\partial a_{i}}, \quad \frac{\partial \Omega_{m}}{\partial T_{n}}=\frac{\partial \Omega_{n}}{\partial T_{m}} .
$$

The prepotential $F$ is defined by the equations

$$
\frac{\partial F}{\partial a_{i}}=\oint_{\beta_{i}} \lambda, \quad \frac{\partial F}{\partial T_{n}}=-2 \pi i \operatorname{res}\left(z^{-n} \lambda\right), \quad \frac{\partial F}{\partial T_{0}}=-2 \pi i \int_{z_{*}=0}^{z=0} \lambda,
$$

where "res" means a residue at $z=0 . \lambda$ then behaves at $\infty$ as

$$
\lambda=\left(-\sum_{n \geq 1} n T_{n} z^{-n-1}+T_{0} z^{-1}-\frac{1}{2 \pi i} \sum_{n \geq 1} \frac{\partial F}{\partial T_{n}} z^{n-1}\right) d z .
$$

Making use of the Riemann bilinear relations it is possible to check integrability conditions (8). For instance

$$
\begin{aligned}
& \frac{\partial}{\partial T_{n}} \frac{\partial F}{\partial a_{i}}=\frac{\partial}{\partial T_{n}} \oint_{\beta_{i}} \lambda=\oint_{\beta_{i}} \Omega_{n} \\
& \quad=-\sum_{j=1}^{r}\left(\oint_{\alpha_{j}} \Omega_{n} \oint_{\beta_{j}} \omega_{i}-\oint_{\alpha_{j}} \omega_{i} \oint_{\beta_{j}} \Omega_{n}\right)
\end{aligned}
$$




$$
\begin{gathered}
=-2 \pi i \operatorname{res}\left(\phi_{n} \omega_{i}\right)=-2 \pi i \operatorname{res}\left(z^{-n} \omega_{i}\right), \\
\frac{\partial}{\partial a_{i}} \frac{\partial F}{\partial T_{n}}=-2 \pi i \frac{\partial}{\partial a_{i}} \operatorname{res}\left(z^{-n} \lambda\right)=-2 \pi i \operatorname{res}\left(z^{-n} \omega_{i}\right),
\end{gathered}
$$

where $\phi_{n}(z)=\int^{z} \Omega_{n}$.

Solutions of the Whitham dynamics eqs.([0)-(10) become relevant to the $N=2$ theory when they obey the homogeneity condition

$$
\sum a_{i} \frac{\partial F}{\partial a_{i}}+T_{0} \frac{\partial F}{\partial T_{0}}+\sum T_{n} \frac{\partial F}{\partial T_{n}}=2 F .
$$

Eq.(10) together with (13) leads to the relation [26

$$
\lambda=\sum_{i=1}^{r} a_{i} \omega_{i}+T_{0} \Omega_{0}+\sum_{n=1} T_{n} \Omega_{n}
$$

The above equation has the form of the meromorphic differentials of $N=2$ theories when $T_{n}$ 's are put to zero except $T_{0}, T_{1}$. Therefore, using the soliton theory we obtain an identity for the prepotential in $N=2$ supersymmetric gauge theories

$$
\sum a_{i} \frac{\partial F}{\partial a_{i}}-2 F=-T_{0} \frac{\partial F}{\partial T_{0}}-T_{1} \frac{\partial F}{\partial T_{1}} .
$$

Evaluation of $T_{0}, T_{1}, \partial F / \partial T_{0}$ is straightforward: we simply read them off from the expansion coefficients of $\lambda$ at $\infty$ in (10). On the other hand, $\partial F / \partial T_{0}$ involves a line integral of the differential $2 \pi i \int_{z_{*}}^{z} \lambda$ which is hard to evaluate in general. This problem is avoided if we consider theories with massless matter fields where the parameter $T_{0}$ vanishes. Therefore in supersymmetric Yang-Mills theories coupled to massless hypermultiplets we obtain a basic formula

$$
\sum a_{i} \frac{\partial F}{\partial a_{i}}-2 F=-T_{1} \frac{\partial F}{\partial T_{1}}=-2 \pi i \operatorname{res}(z \lambda) \operatorname{res}\left(z^{-1} \lambda\right)
$$

We shall show that the right-hand-side of eq.(16) has the universal form

$$
8 \pi i b_{1} u
$$

in all the examples discussed below. Note that in $S O\left(N_{c}\right)$ gauge theories coupled to the vector matter $T_{0}$ vanishes due to symmetry $x \rightarrow-x$ of the curve. 
We now compute the right-hand-side of eq.(16) in $N=2$ theories where curves and differentials are known explicitly.

$S U\left(N_{c}\right)$ theory with matter in the vector representation [18]

(a) $N_{f}<N_{c}$

$$
\begin{aligned}
y^{2} & =C(x)^{2}-\Lambda_{N_{f}}^{2 N_{c}-N_{f}} G(x), \\
C(x) & =x^{N_{c}}-\sum_{i=2}^{N_{c}} u_{i} x^{N_{c}-i}, \quad G(x)=\prod_{i=1}^{N_{f}}\left(x+m_{i}\right), \quad u=u_{2} \\
\lambda & =\frac{x d x}{2 \pi i y}\left(\frac{C G^{\prime}}{2 G}-C^{\prime}\right), \quad z=1 / x \\
& \approx \frac{d z}{2 \pi i}\left(\left(\frac{N_{f}}{2}-N_{c}\right) z^{-2}-\frac{1}{2} \sum_{i=1}^{N_{f}} m_{i} z^{-1}+\left(-2 u+\frac{1}{2} \sum_{i=1}^{N_{f}} m_{i}^{2}\right)\right) .
\end{aligned}
$$

Thus

$$
T_{1}=\frac{-N_{f} / 2+N_{c}}{2 \pi i}, \quad T_{0}=-\frac{1}{4 \pi i} \sum_{i=1}^{N_{f}} m_{i}, \quad \frac{\partial F}{\partial T_{1}}=2 u-\frac{1}{2} \sum_{i=1}^{N_{f}} m_{i}^{2}
$$

and we reproduce (11) in the massless limit $\left(b_{1}=\left(2 N_{c}-N_{f}\right) / 16 \pi^{2}\right)$.

(b) $N_{f} \geq N_{c}$

$$
\begin{aligned}
& y^{2}=F(x)^{2}-\Lambda_{N_{f}}^{2 N_{c}-N_{f}} G(x), \\
& F(x)=C(x)+\frac{\Lambda_{N_{f}}^{2 N_{c}-N_{f}}}{4} \sum_{i=0}^{N_{f}-N_{c}} x^{N_{f}-N_{c}-i} t_{i}(m), \\
& t_{k}(m)=\sum_{i_{1}<\cdots<i_{k}} m_{i_{1}} \cdots m_{i_{k}} .
\end{aligned}
$$

We again find eq.(1) in the massless limit.

$S O\left(N_{c}\right)$ gauge theory with matter in the vector representation [22]

(a) $N_{f}<N_{c} / 2-2$ for $N_{c}$ even, $N_{f}<N_{c} / 2-3 / 2$ for $N_{c}$ odd 


$$
\begin{aligned}
& y^{2}=C(x)^{2}-\Lambda_{N_{f}}^{2\left(N_{c}-2-N_{f}\right)} G(x), \\
& C(x)= \begin{cases}\prod_{i=1}^{N_{c} / 2}\left(x^{2}-e_{i}^{2}\right)=x^{N_{c}}-u x^{N_{c}-2}-\cdots, & N_{c} \text { even } \\
\prod_{i=1}^{\left(N_{c}-1\right) / 2}\left(x^{2}-b_{i}^{2}\right)=x^{N_{c}-1}-u x^{N_{c}-3}-\cdots, \quad N_{c} \text { odd }\end{cases} \\
& G(x)=x^{d} \prod_{i=1}^{N_{f}}\left(x^{2}-m_{i}^{2}\right), \quad \begin{cases}d=4 & \text { for } N_{c} \text { even } \\
d=2 & \text { for } N_{c} \text { odd }\end{cases} \\
& \lambda=\frac{x d x}{2 \pi i y}\left(\frac{C G^{\prime}}{2 G}-C^{\prime}\right) .
\end{aligned}
$$

(b) $N_{f} \geq N_{c} / 2-2$ for $N_{c}$ even, $\quad N_{f} \geq N_{c} / 2-3 / 2$ for $N_{c}$ odd

$$
\begin{aligned}
y^{2} & =\left(C(x)+\Lambda^{2\left(N_{c}-N_{f}-2\right)} P(x)\right)^{2}-\Lambda_{N_{f}}^{2\left(N_{c}-2-N_{f}\right)} G(x), \\
\lambda & =\frac{x d x}{2 \pi i y}\left(\frac{C G^{\prime}}{2 G}-C^{\prime}\right) .
\end{aligned}
$$

Here $P(x)$ is a polynomial of order $2 N_{f}-\left(N_{c}-2\right)$ in $x$ and $m_{i}$. We note that in $S O\left(N_{c}\right)$ gauge theories $2 r$ periods out of $2(2 r-1)$ periods of $\Sigma$ are independent due to $x \rightarrow-x$ symmetry of the curve. In both cases (a), (b) we find

$$
T_{0}=0, \quad T_{1}=\frac{-N_{f}+N_{c}-2}{2 \pi i}, \quad \frac{\partial F}{\partial T_{1}}=2 u-\sum_{i=1}^{N_{f}} m_{i}^{2} .
$$

Thus eq.(1) holds also in $S O\left(N_{c}\right)$ theories $\left(b_{1}=2\left(N_{c}-2-N_{f}\right) / 16 \pi^{2}\right)$.

So far we have derived eq.(11) only in the case of $S U\left(N_{c}\right)$ and $S O\left(N_{c}\right)$ gauge theories with matter in the vector representations for which data on algebraic curves and differential forms are available. However, it seems natural to conjecture that it holds in the Coulomb branch of all $N=2$ Yang-Mills theories with arbitrary gauge groups and arbitrary massless hypermultiplet representations. In order to discuss general cases, deeper understanding of the choice of cycles and sub-spaces of Jacobians of $\Sigma$ seems necessary [25]. 
Equation (11) will play a basic role when 4 dimensional gauge theories are embedded into supergravity and superstring theories. In locally supersymmetric theories the variable $T_{1}$ will be identified as the expectation value of the dilaton field as in the important examples worked out in ref.[32]. $T_{n}$ variables in eq. (13) restore the homogeneity of the prepotential destroyed by the non-vanishing $\beta$-function and bring eq.(13) into a form known in the special geometry of $N=2$ supergravity. It will be very interesting to see if it is possible to provide physical interpretation of the $T_{n}$ variables which may describe the gravity sector of $N=2$ supergravity theories. Eq.(13) is also reminiscent of the Virasoro $L_{0}$ condition in 2 dimensional topological $\sigma$ model coupled to topological gravity [33]. It is interesting to see if there are analogues of $W$ conditions in the case of higher rank $N=2$ gauge theories.

We would like to thank Dr. T. Nakatsu for discussions on his work. Research of T.E. and S.K.Y. is supported in part by the Grant-in-Aid for Scientific Research on Priority Area 213 "Infinite Analysis", Japan Ministry of Education. 


\section{References}

[1] A. Sen, Int. J. Mod. Phys. A9 (1994) 3007.

[2] N. Seiberg and E. Witten, Nucl. Phys. B426 (1994) 19.

[3] N. Seiberg and E. Witten, Nucl. Phys. B431 (1994) 484.

[4] C. Vafa and E. Witten, Nucl. Phys. B431 (1994) 3.

[5] C.M. Hull and P.K. Townsend, Nucl. Phys. B438 (1995) 109.

[6] E. Witten, Nucl. Phys. B443 (1995) 85.

[7] J. Harvey and A. Strominger, Nucl. Phys. B449 (1995) 535.

[8] A. Sen, String-String Duality Conjecture in Six Dimensions and Charged Solitonic Strings, hep-th/9504027.

[9] A. Strominger, Nucl. Phys. B451 (1995) 96.

[10] S. Kachru and C. Vafa, Nucl. Phys. B450 (1995) 69.

[11] S. Ferrara, J. Harvey, A. Strominger and C. Vafa, Second-Quantized Mirror Symmetry, hep-th/9505162.

[12] E. Witten, Some Comments on String Dynamics, hep-th/9507121.

[13] J.H. Schwarz, The Power of M Theory, hep-th/9510086.

[14] P. Argyres and A. Faraggi, Phys. Rev. Lett. 74 (1995) 3931.

[15] A. Klemm, W. Lerche, S. Theisen and S. Yankielowicz, Phys. Lett. B344 (1995) 169.

[16] U.H. Danielsson and B. Sundborg, The Moduli Space and Monodromies of $N=2$ Supersymmetric $S O(2 r+1)$ Yang-Mills Theory, hepth9504102 .

[17] A. Brandhuber and K. Landsteiner, Phys. Lett. B358 (1995) 73.

[18] A. Hanany and Y. Oz, Nucl. Phys. B452 (1995) 283. 
[19] P. Argyres, R. Plesser and A. Shapere, Phys. Rev. Lett. 75 (1995) 1699.

[20] J.A. Minahan and D. Nemeschansky, Hyperelliptic Curves for Supersymmetric Yang-Mills, hep-th/9507032.

[21] P.C. Argyres and A.D. Shapere, The Vacuum Structure Of $N=2$ Super-QCD With Classical Gauge Groups, hep-th/9509175.

[22] A. Hanany, On The Quantum Moduli Space of Vacua of $N=2$ Supersymmetric Gauge Theories, hep-th/9509176.

[23] G.B. Whitham, Proc. Roy. Soc. A283 (1965) 238.

[24] A. Gorsky, I. Krichever, A. Marshakov, A. Mironov and A. Morozof, Phys. Lett. B355 (1995) 466.

[25] E. Martinec and N. Warner, Integrable Systems and Supersymmetric Gauge Theory, hep-th/9509161.

[26] T. Nakatsu and K. Takasaki, Whitham-Toda Hierarchy and $N=2$ Supersymmetric Yang-Mills Theory, hep-th/9509162.

[27] M. Matone, Phys. Lett. B357 (1995) 342.

[28] J. Sonnenschein, S. Theisen and S. Yankielowicz, On the Relation Between the Holomorphic Prepotentials and the Quantum Moduli in SUSY Gauge Theories, hep-th/9510129.

[29] A. Klemm, W. Lerche and S. Theisen, Nonperturbative Effective Actions of N=2 Supersymmetric Gauge Theories, hep-th/9505150.

[30] A. Ceresole, R. D’Auria and S. Ferrara, Phys. Lett. B339 (1995) 71.

[31] K. Ito and S.-K. Yang, Prepotentials in N=2 SU(2) Supersymmetric Yang-Mills Theory with Massless Hypermultiplets, hep-th/9507144.

[32] S. Kachru, A. Klemm, W. Lerche, P. Mayr and C. Vafa, Nonperturbative Results on the Point Particle Limit of $N=2$ Heterotic String Compactification, hep-th/9508155.

[33] K. Hori, Nucl. Phys. B439 (1995) 395. 